\title{
Searching for Possible Siblings of the Sun from a Common Cluster Based on Stellar Space Velocities
}

\author{
V.V. Bobylev ${ }^{1,2}$, A.T. Bajkova ${ }^{1}$, A. Mylläri ${ }^{3}$, and M. Valtonen ${ }^{4}$ \\ 1 Pulkovo Astronomical Observatory, Russian Academy of Sciences, Pulkovskoe sh. 65, St. \\ Petersburg, 196140 Russia; E-mail: vbobylev@gao.spb.ru \\ 2 Sobolev Astronomical Institute, St. Petersburg State University, Universitetskii pr. 28, \\ Petrodvorets, 198504 Russia \\ 3 Abo Akademi University, Turku, Finland \\ ${ }^{4}$ Helsinki Institute of Physics, Helsinki University, Finland
}

\begin{abstract}
We propose a kinematic approach to searching for the stars that could be formed with the Sun in a common "parent" open cluster. The approach consists in preselecting suitable candidates by the closeness of their space velocities to the solar velocity and analyzing the parameters of their encounters with the solar orbit in the past in a time interval comparable to the lifetime of stars. We consider stars from the Hipparcos catalog with available radial velocities. The Galactic orbits of stars have been constructed in the Allen-Santillan potential by taking into account the perturbations from the spiral density wave. We show that two stars, HIP 87382 and HIP 47399, are of considerable interest in our problem. Their orbits oscillate near the solar orbit with an amplitude of $\approx 250$ pc; there are short-term close encounters to distances $<10$ pc. Both stars have an evolutionary status and metallicity similar to the solar ones.
\end{abstract}

\section{INTRODUCTION}

According to present-day observations, stars are formed in groups or clusters of various strengths. It is highly likely that the Sun was formed in some "parent" open star cluster.

Comparison of the solar chemical composition with the metallicity distribution in the Galactic disk led to the conclusion that the Sun was born $\approx 2 \mathrm{kpc}$ closer to the Galactic center relative to its current position (Wielen et al. 1996). A discussion of this problem in light of the currently available data can be found in Acharova et al. (2010). Revealing possible siblings of the Sun will allow a better understanding of both the mechanisms of radial migration in the Galactic disk (Minchev and Famaey 2010; Shevchenko 2010) and the conditions under which the Sun was formed (Williams 2010). It is hypothesized that the stars born together retain their chemical homogeneity for a very long time (BlandHawthorn and Freeman 2004; Bland-Hawthorn et al. 2010).

Therefore, as a method of searching for such stars, these authors suggested analyzing spectroscopic data to determine the abundances of various elements. In particular, the 
spectroscopic method has been successfully used to prove the dynamical origin of the stars belonging to the Hyades cluster (Pompéia ia et al. 2011).

Obviously, the stars formed together with the Sun are also of great interest in studying both the dynamical evolution of the Solar system and the development of terrestrial life (Valtonen et al. 2009).

The difficulties of revealing such stars in the solar neighborhood are associated with the Sun's great age $(\approx 4.6 \mathrm{Gyr})$, the dynamical evolution of open star clusters (OSCs) during this time, and a large uncertainty in Galactic parameters. Numerical simulations of the dynamical evolution of OSCs show (Chumak et al. 2005; Chumak and Rastorguev 2006a, 2006b) that stellar tails stretched along the Galactic cluster orbits develop in them with time. The OSC remnants existing in the form of tails must completely dissolve and mix with the stellar background in a time $\approx 2$ Gyr (Küpper et al. 2008). According to the estimates by Portegies Zwart (2009), 10-60 stars from the parent (for the Sun) OSC containing $\approx 10^{3}$ members can now be in a solar neighborhood about $100 \mathrm{pc}$ in radius.

The attempts at searching for the Sun's siblings from a common cluster are known in the literature. For example, Brown et al. (2010) searched for suitable candidates among the stars of the Hipparcos catalog (1997) in an updated version (van Leeuwen 2007). A list of candidates containing six stars was proposed. However, when the orbital motion of stars was simulated, the influence of the perturbations from the spiral density wave was disregarded. In addition, the search was carried out using only the stellar parallaxes and proper motions (without radial velocities). Mishurov and Acharova (2010) showed that the influence of the spiral density wave in a time interval of 4.6 Gyr leads to a significant dispersal of the members of an initially compact cluster in both radial and tangential directions. For about a hundred dispersed stars to be now observable in a solar neighborhood $\approx 100 \mathrm{pc}$ in radius, the parent cluster must contain $\approx 10^{4}$ members.

In this paper, we suggest applying a kinematic approach to searching for the stars that could be formed with the Sun in a common parent cluster. This approach consists in (1) selecting suitable candidates by the closeness of their space velocities to the solar velocity, (2) constructing the stellar and solar orbits in the Galactic potential including the perturbations from the spiral density wave, and (3) analyzing the parameters of the encounters between the stellar orbits and the solar orbit in the past in a time interval comparable to the lifetime of stars.

\section{DATA}

The initial set of kinematic data on the stars from the Hipparcos catalog (1997) that we used is described in detail in Bobylev et al. (2010). It contains the parallaxes and proper motions of about 35000 stars taken from a revised version of the Hipparcos catalog (van Leeuwen 2007). The radial velocities from the PCRV catalog (Gontcharov 2006) are available for each star.

We selected $162 \mathrm{~F}, \mathrm{G}$, and $\mathrm{K}$ stars with a relative parallax error $\sigma_{\pi} / \pi<15 \%$ and magnitude of the total stellar space velocity $(U, V, W)$ relative to the Sun $\sqrt{U^{2}+V^{2}+W^{2}}<$ $8 \mathrm{~km} \mathrm{~s}^{-1}$, where $8 \mathrm{~km} \mathrm{~s}^{-1}$ was estimated from a typical random error in each of the velocities $U, V, W$, which are $\approx 2 \mathrm{~km} \mathrm{~s}^{-1}$. Note also that age and metallicity estimates are 
Table 1: Parameters of he model Galactic potential

\begin{tabular}{|c|c|}
\hline$M_{C}$ & $606 \mathrm{M}_{G}$ \\
\hline$M_{D}$ & $3690 \mathrm{M}_{G}$ \\
\hline$M_{H}$ & $4615 \mathrm{M}_{G}$ \\
\hline$b_{C}$ & $0.3873 \mathrm{kpc}$ \\
\hline$a_{D}$ & $5.3178 \mathrm{kpc}$ \\
\hline$b_{D}$ & $0.25 \mathrm{kpc}$ \\
\hline$a_{H}$ & $12 \mathrm{kpc}$ \\
\hline
\end{tabular}

available for a significant fraction of the selected stars (Holmberg et al. 2009).

Remarkably, two of the six candidates from Brown et al. (2010) entered into our list of 162 stars: HIP 21158 and HIP 99689.

\section{THE CONSTRUCTION OF ORBITS}

We calculated the stellar and solar orbits by solving the following system of equations of motion based on a realistic model of the Galactic gravitational potential (Fernandez et al. 2008):

$$
\begin{gathered}
\ddot{\xi}=-\frac{\partial \Phi}{\partial \xi}-\Omega_{0}^{2}\left(R_{0}-\xi\right)-2 \Omega_{0} \dot{\eta}, \\
\ddot{\eta}=-\frac{\partial \Phi}{\partial \eta}+\Omega_{0}^{2} \eta+2 \Omega_{0} \dot{\xi}, \\
\ddot{\zeta}=-\frac{\partial \Phi}{\partial \zeta},
\end{gathered}
$$

where $\Phi$ is the Galactic gravitational potential; the $(\xi, \eta, \zeta)$ coordinate system with the center at the Sun rotates around the Galactic center with a constant angular velocity $\Omega_{0}$, with the $\xi, \eta$, and $\zeta$ axes being directed toward the Galactic center, in the direction of Galactic rotation, and toward the Galactic North Pole, respectively; $R_{0}$ is the Galactocentric distance of the Sun.

We used the Allen-Santillan (1991) model Galactic potential. The system of equations (1) was solved numerically by the fourth-order Runge-Kutta method.

In the Allen-Santillan (1991) model, the Galactocentric distance of the Sun is taken to be $R_{0}=8.5 \mathrm{kpc}$ and the circular velocity of the Sun around the Galactic center is $V_{0}=\left|\Omega_{0}\right| R_{0}=220 \mathrm{~km} \mathrm{~s}^{-1}$. The axisymmetric Galactic potential is represented as the sum of three components - the central (bulge), disk, and halo ones:

$$
\Phi=\Phi_{C}+\Phi_{D}+\Phi_{H} .
$$

The central component of the Galactic potential in cylindrical coordinates $(r, \theta, z)$ is represented as

$$
\Phi_{C}=-\frac{M_{C}}{\left(r^{2}+z^{2}+b_{C}^{2}\right)^{1 / 2}},
$$


where $M_{C}$ is the mass and $b_{C}$ is the scale parameter. The disk component is

$$
\Phi_{D}=-\frac{M_{D}}{\left\{r^{2}+\left[a_{D}+\left(z^{2}+b_{D}^{2}\right)^{1 / 2}\right]^{1 / 2}\right\}^{1 / 2}},
$$

where $M_{D}$ is the mass, $a_{D}$ and $b_{D}$ are the scale parameters. The halo component is

$$
\Phi_{H}=-\frac{M(R)}{R}-\int_{R}^{100} \frac{1}{R^{\prime}} \frac{d M\left(R^{\prime}\right)}{d R^{\prime}} d R^{\prime},
$$

where

$$
M(R)=\frac{M_{H}\left(R / a_{H}\right)^{2.02}}{1+\left(R / a_{H}\right)^{1.02}},
$$

Here, $M_{H}$ is the mass and $a_{H}$ is the scale parameter. If $R$ is measured in kpc and $M_{C}, M_{D}, M_{H}$ are measured in units of the Galactic mass $\left(M_{G}\right)$ equal to $2.32 \times 10^{7} M_{\odot}$, then the Gravitational constant is $G=1$ and the unit of measurement of the potential $\Phi$, along with the individual components of (3)-(5), is $100 \mathrm{~km}^{2} \mathrm{~s}^{-2}$.

All of the Allen-Santillan model parameters adopted here are given in Table 1.

If the spiral density wave is taken into account (Lin and Shu 1964; Lin et al. 1969), then the following term is added to the right-hand side of Eq. (2) (Fernandez et al. 2008):

$$
\Phi_{s p}(R, \theta, t)=A \cos \left[m\left(\Omega_{p} t-\theta\right)+\chi(R)\right]
$$

where

$$
\begin{gathered}
A=\frac{\left(R_{0} \Omega_{0}\right)^{2} f_{r 0} \tan i}{m}, \\
\chi(R)=-\frac{m}{\tan i} \ln \left(\frac{R}{R_{0}}\right)+\chi_{\odot} .
\end{gathered}
$$

Here, $A$ is the amplitude of the spiral wave potential; $f_{r 0}$ is the ratio of the radial component of the perturbation from the spiral arms to the Galaxy's total attraction; $\Omega_{p}$ is the pattern speed of the wave; $m$ is the number of spiral arms; $i$ is the arm pitch angle, $i<0$ for a winding pattern; $\chi$ is the phase of the radial wave (the arm center then corresponds to $\left.\chi=0^{\circ}\right)$; and $\chi_{\odot}$ is the Sun's phase in the spiral wave.

The spiral wave parameters are very unreliable (a review of the problem can be found in Fernandez et al. (2001) and Gerhard (2010)). The simplest model of a two-armed spiral pattern is commonly used, although, as analysis of the spatial distribution of young Galactic objects (young stars, star-forming regions, or hydrogen clouds) shows, both threeand four-armed patterns are possible (Russeil 2003; Englmaier et al. 2008; Hou et al. 2009). More complex models are also known, for example, the kinematic model by Lépine et al. (2001) that combines two- and four-armed spiral patterns rigidly rotating with an angular velocity close $\Omega_{0}$. Note also the spiral ring Galactic model (Mel'nik and Rautiainen 2009) that includes two outer rings elongated perpendicular and parallel to the bar, an inner ring elongated parallel to the bar, and two small fragments of spiral arms. As applied to the Galaxy, the theories of nonstationary spiral waves with a fairly short stationarity time (several $100 \mathrm{Myr}$ ), a variable rotation rate, and a variable number of arms are also considered (Sellwood and Binney 2002; Baba et al. 2009). The currently 
available data do not yet allow one of the listed models to be unequivocally chosen. Therefore, here we apply the model of a stationary spiral pattern with different numbers of spiral arms.

Given the data on the Galactic bar rotation (Debattista et al. 2002), the pattern speed $\Omega_{p}$ can lie within the range $15-65 \mathrm{~km} \mathrm{~s}^{-1} \mathrm{kpc}^{-1}$. The possibility of the coexistence of several pattern speeds, a rapidly rotating bar and a slower spiral pattern, is also considered (Minchev and Famaey 2010; Gerhard 2010). Here, we disregard the influence of the bar. Therefore, we choose $\Omega_{p}$ from the range 15-30 $\mathrm{km} \mathrm{s}^{-1} \mathrm{kpc}^{-1}$ (Popova and Loktin 2005; Naoz and Shaviv 2007; Gerhard 2010).

The pitch angle $i$ is known relatively well and is $-5^{\circ} \div-7^{\circ}$ and $-10^{\circ} \div-14^{\circ}$ for the two- and four-armed spiral patterns, respectively.

The amplitudes of the velocities of the perturbation from the spiral density wave are $5-10 \mathrm{~km} \mathrm{~s}^{-1}$ (Mishurov and Zenina 1999; Fernandez et al. 2001; Bobylev and Bajkova 2010) in both tangential and radial directions.

The Sun's phase in the wave $\chi_{\odot}$ is known with a very large uncertainty. For example, according to Fernandez et al. (2001), this angle lies within the range $284^{\circ}-380^{\circ}$. Having analyzed the kinematics of OSCs (Bobylev et al. 2008; Bobylev and Bajkova 2010), we found $\chi_{\odot}$ close to $-117^{\circ}$ (or $243^{\circ}$ ), while the data on masers yielded an estimate of $\chi_{\odot}=-130 \pm 10^{\circ}$. (Bobylev and Bajkova 2010); here, the minus implies that hat we measure the phase angle from the center of the Carina-Sagittarius spiral arm. From the position of the Sun among the spiral arms, it is obvious that this angle is close to $-\pi / 2$ (Russeil 2003).

According to the classical approach in the linear density-wave theory (Yuan 1969), the ratio $f_{r 0}$ lies within the range $0.04-0.07$ and the most probable value is $f_{r 0}=0.05$. The upper limit $f_{r 0}=0.07$ is determined by the velocity dispersion of young objects observed in the Galaxy (at $f_{r 0}=0.07$, the dispersion must reach $25 \mathrm{~km} \mathrm{~s}^{-1}$, which exceeds a typical observed value of $10-15 \mathrm{~km} \mathrm{~s}^{-1}$ ).

We adopted the following parameters: the two-armed spiral pattern $(m=2)$, the pattern speed of the spiral wave $\Omega_{p}=20 \mathrm{~km} \mathrm{~s}^{-1} \mathrm{kpc}^{-1}$, the pitch angle $i=-5^{\circ}$, the ratio $f_{r 0}=0.05$, and the Sun's phase in the wave $\chi_{\odot}=-117^{\circ}$. We also took into account the Sun's displacement from the Galactic plane $Z_{\odot}=17 \mathrm{pc}$ (Joshi 2007) and used the present-day peculiar velocity of the Sun relative to the local standard of rest $\left(U_{\odot}, V_{\odot}, W_{\odot}\right)_{L S R}=(10,11,7) \mathrm{km} \mathrm{s}^{-1}$ (Binney 2010; Schönrich et al. 2010; Bobylev and Bajkova 2010).

For all of the selected 162 stars, we determined the relative distances $d$ between the star and the Sun as well as their velocity difference $d V$ in the time interval in the past with a boundary of -4.5 Gyr. The minimum values of these parameters, $d_{\min }$ and $d V_{\min }$, were also determined for a certain time $t_{\min }$.

\section{RESULTS}

\section{Encounter Parameters}

Figure 1 presents the $(B-V)$ color-absolute magnitude $M_{V}$ diagram for the 162 selected candidates. To calculate $M_{V}$, we used the apparent $V$ magnitudes from the Hipparcos 
Table 2: Data on the stars

\begin{tabular}{|c|c|c|c|c|c|c|c|}
\hline HIP & SP & Age, Gyr & {$[\mathrm{Fe} / \mathrm{H}]$} & Reference & $d_{\text {min }}, \mathrm{pc}$ & $t_{\min }, \mathrm{Gyr}$ & $d V_{\min }, \mathrm{km} \mathrm{s}^{-1}$ \\
\hline 47399 & F8V & $4.3(0-7.3)$ & -0.21 & $(1)$ & 1 & -3.5 & 2 \\
& & & -0.10 & $(2)$ & & & \\
\hline 87382 & F8V & $3.5(2.6-4.0)$ & +0.04 & $(1)$ & 4 & -3.1 & 6 \\
& & $3.7(3.2-4.2)$ & +0.01 & $(3,2)$ & & & \\
\hline
\end{tabular}

Note. For the age, the upper and lower limits of the estimate calculated with a 1 error are given in parentheses; 1 -Holmberg et al. (2009), where the metallicity was determined from Strömgren photometry; 2-Robinson et al. (2007), where the metallicity was determined from Lick indices; 3 - Takeda et al. (2007).

catalog. We see from Fig. 1 that the number of candidates lying close to the isochrones and having nearly solar spectral types will be considerably smaller than the total number of stars selected by their kinematics at the first step.

The distributions of the sample of 162 stars in the Galactic $X Y, Z X$, and $Y Z$ coordinate planes are shown in Fig. 2. The $X, Y$, and $Z$ coordinate axes are directed toward the Galactic center, in the direction of Galactic rotation, and toward the Galactic Pole, respectively.

We found that only two stars from our sample, namely HIP 47399 (Fig. 3) and HIP 87382 (Fig. 4), can be acceptable candidates. For them, there is good agreement between their estimated ages and the kinematic encounter parameters $(d, d V)$ found. In Figs. 1 and 2, the positions of these stars are marked by the large filled circles (the random error bars are within the circles) and the crosses, respectively.

For HIP 47399, we have the following initial data: $\alpha=9^{h} 39^{m} 27^{s} .4, \delta=42^{\circ} 17^{\prime} 09^{\prime \prime}$, $\mu_{\alpha} \cos \delta=-4.29 \pm 1.38 \operatorname{mas~yr}^{-1}, \mu_{\delta}=-6.70 \pm 0.59 \operatorname{mas~yr}^{-1}, \pi=13.87 \pm 0.95$ mas (the heliocentric distance $r=72 \pm 5 \mathrm{pc}$ ), the radial velocity $V_{r}=-7.3 \pm 3.4 \mathrm{~km} \mathrm{~s}^{-1}$, $U=3.9 \pm 2.3 \mathrm{~km} \mathrm{~s}^{-1}, V=-2.5 \pm 0.3 \mathrm{~km} \mathrm{~s}^{-1}, W=-6.3 \pm 2.6 \mathrm{~km} \mathrm{~s}^{-1}$.

For HIP 87382: $\alpha=17^{h} 51^{m} 14^{s} .0, \delta=40^{\circ} 04^{\prime} 20^{\prime \prime}, \mu_{\alpha} \cos \delta=-16.86 \pm 0.28 \mathrm{mas} \mathrm{yr}^{-1}$, $\mu_{\delta}=11.01 \pm 0.42 \mathrm{mas} \mathrm{yr}^{-1}, \pi=29.76 \pm 0.36 \operatorname{mas}(r=33.6 \pm 0.4 \mathrm{pc}), V r=1.6 \pm 0.2 \mathrm{~km}$ $\mathrm{s}^{-1}, U=-1.0 \pm 0.1 \mathrm{~km} \mathrm{~s}^{-1}, V=0.4 \pm 0.2 \mathrm{~km} \mathrm{~s}^{-1}, W=3.4 \pm 0.1 \mathrm{~km} \mathrm{~s}^{-1}$. The ages and metallicities of these stars estimated by various authors are given in Table 2. The error in $[\mathrm{Fe} / \mathrm{H}]$ is typically $0.1-0.15$ dex. This suggests that the metallicity of HIP 47399 and HIP 87382 is nearly solar, within the error limits.

HIP 21158 and HIP 99689 common to the list by Brown et al. (2010) do not withstand the described test for close encounters in the past.

The encounter parameters $d$ and $d V$ depend on the adopted components of the Suns peculiar velocity vector $\left(U_{\odot}, V_{\odot}, W_{\odot}\right)_{L S R}$ and on whether or not we take into account the influence of the spiral wave. Without allowance for the spiral wave, there are no close encounters between HIP 47399 and the Sun, while the closest encounters for HIP 87382 $\left(d_{\text {min }}=16 \mathrm{pc}, d V_{\text {min }}=16 \mathrm{~km} \mathrm{~s}^{-1}\right)$ occur at $t_{\min }=-1.2$ Gyr. It may be concluded that allowance for the spiral density wave has a significant influence on the results. 


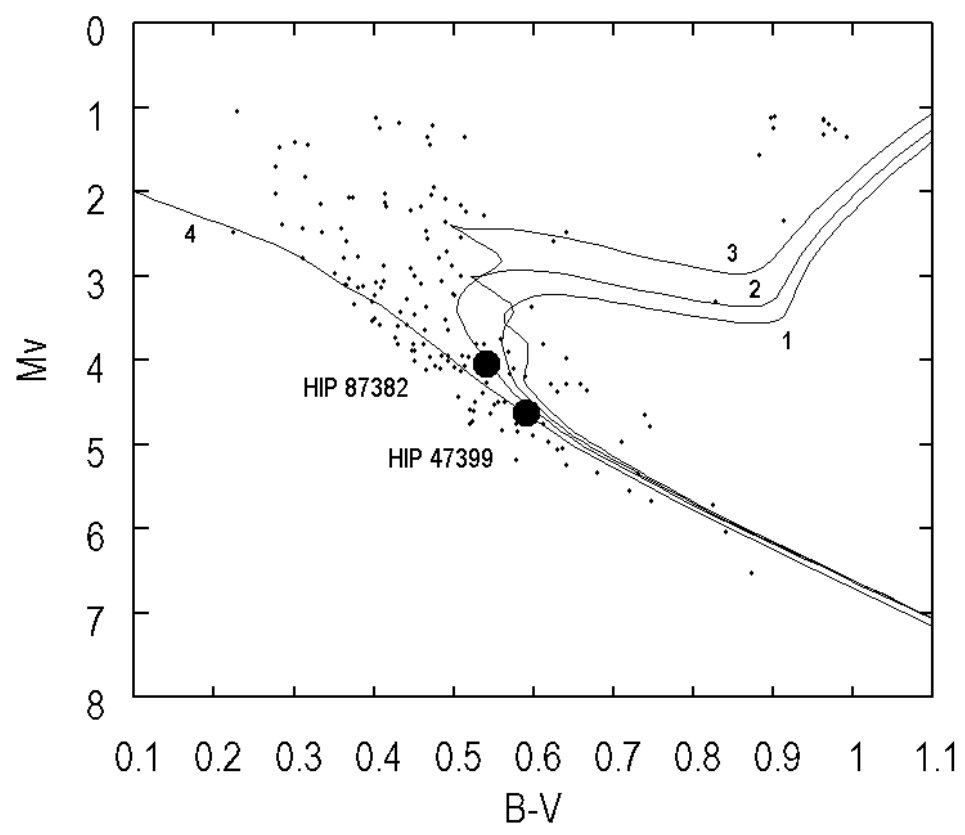

Figure 1: Positions of stars on the colorabsolute magnitude diagram; the zero-age main sequence (4) and three isochrones (Demarque et al. 2004) for ages of 3 (3), 4 (2), and 5 (1) Gyr are marked.

The encounter parameters calculated for HIP 47399 and HIP 87382 by taking into account the influence of the spiral wave are given in the last columns of Table 2.

\section{The Probability of Close Encounters}

To estimate the probability that a star was born together with the Sun in a common star cluster, we should take into account the following. The lifetime of the OSC as a gravitationally bound structure is typically no more than 2 Gyr. The OSC core size is typically about $10 \mathrm{pc}$. The OSC has an open halo and a tail elongated along its orbit develops with time. We assumed that the OSC was born at a time of -4.5 Gyr.

We then consider the probability $(p)$ that the star and the Sun belong to the OSC core to be nonzero if the following conditions are met for them in the time interval $-4.5<t<-2.5$ Gyr: the relative distance along the Galactic radius is $|d R|<20 \mathrm{pc}$, along the vertical axis is $|d Z|<20 \mathrm{pc}$, and along the orbit is $|d Y|<20 \mathrm{pc}$, then $d=$ $\sqrt{d R^{2}+d Z^{2}+d Y^{2}}<35$ pc. For HIP 87382, according to the upper panel in Fig. 4, these conditions are close to fulfilment in this time interval - we observe a minimum of $d=40-60$ pc near -3.5 Gyr. Similar reasoning is also valid for HIP 47399.

As a result, we calculate $p$ as the ratio of the number of points in the narrow peaks (Figs. 3 and 4) satisfying the adopted constraints to the total number of points in a given time interval.

The constraint on the relative velocity at the encounter time should also be taken into account. Then, for example, for HIP 87382 at $d<10 \mathrm{pc}$ and $|d V|<10 \mathrm{~km} \mathrm{~s}^{-1}$ 

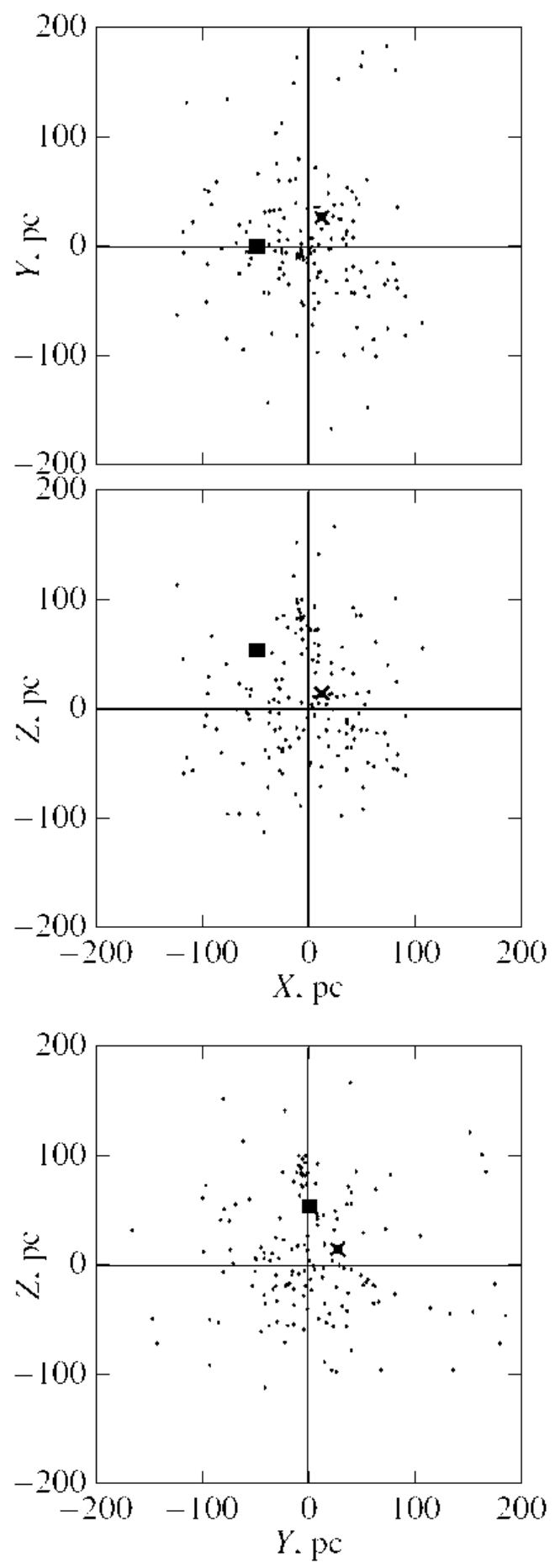

Figure 2: Distributions of stars in the $X Y, Z X$, and $Y Z$ planes; the filled square and cross mark the stars HIP 47399 and HIP 87382, respectively. 


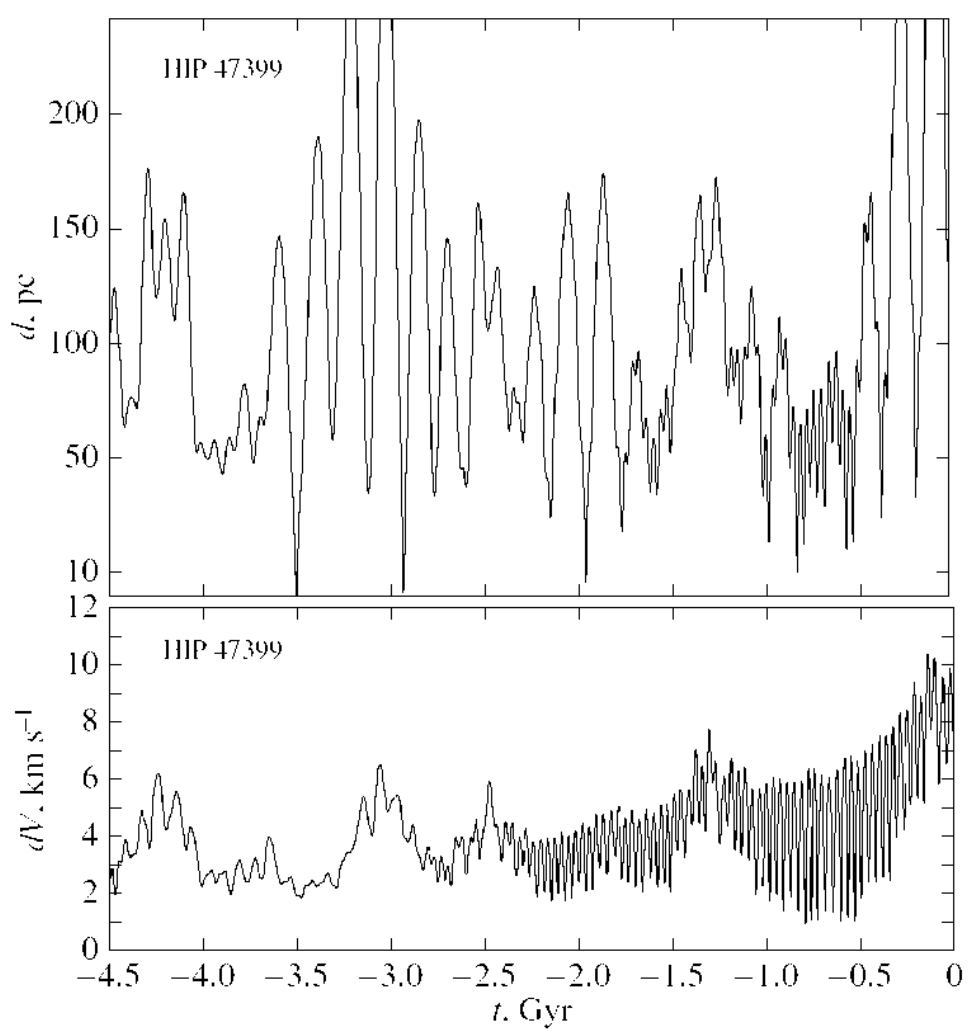

Figure 3: Parameters $d$ and $d V$ of the encounter between HIP 47399 and the solar orbit versus time for the two-armed spiral pattern.

in the time interval from -4.5 to $-2.5 \mathrm{Gyr}$, the probability $p=0.001(0.1 \%)$ is then a more rigorous estimate. For less rigorous requirements, $d<20 \mathrm{pc}$ and $|d V|<10 \mathrm{~km} \mathrm{~s}^{-1}$, $p=0.006(0.6 \%)$.

For HIP 47399, these numbers are slightly larger (because its relative velocity is always low, $d V<10 \mathrm{~km} \mathrm{~s}^{-1}$, as we see from the lower panel in Fig. 3): $p=0.006(0.6 \%)$ for $d<10 \mathrm{pc}$ and $|d V|<10 \mathrm{~km} \mathrm{~s}^{-1}$ and $p=0.014(1.4 \%)$ for $d<20 \mathrm{pc}$ and $|d V|<10 \mathrm{~km}$ $\mathrm{s}^{-1}$.

For a rigorous estimation of $p$, we should take into account the errors in the observational data and the errors in the parameters of the Suns peculiar velocity and the parameters of the spiral density wave using the method of statistical simulations. In Fig. 5, the parameters of the mutual encounters between HIP 87382 and HIP 87382 are plotted against time. We can see that their relative velocity in the time interval from -3.5 to $-2.8 \mathrm{Gyr}$ is $5-10 \mathrm{~km} \mathrm{~s}^{-1}$; there is a peak encounter to distances of less than 10 pc. Hence it may be concluded that within the framework of the approach used, three objects (the Sun, HIP 47399, and HIP 87382) are possible candidates for being members of a common open cluster. 


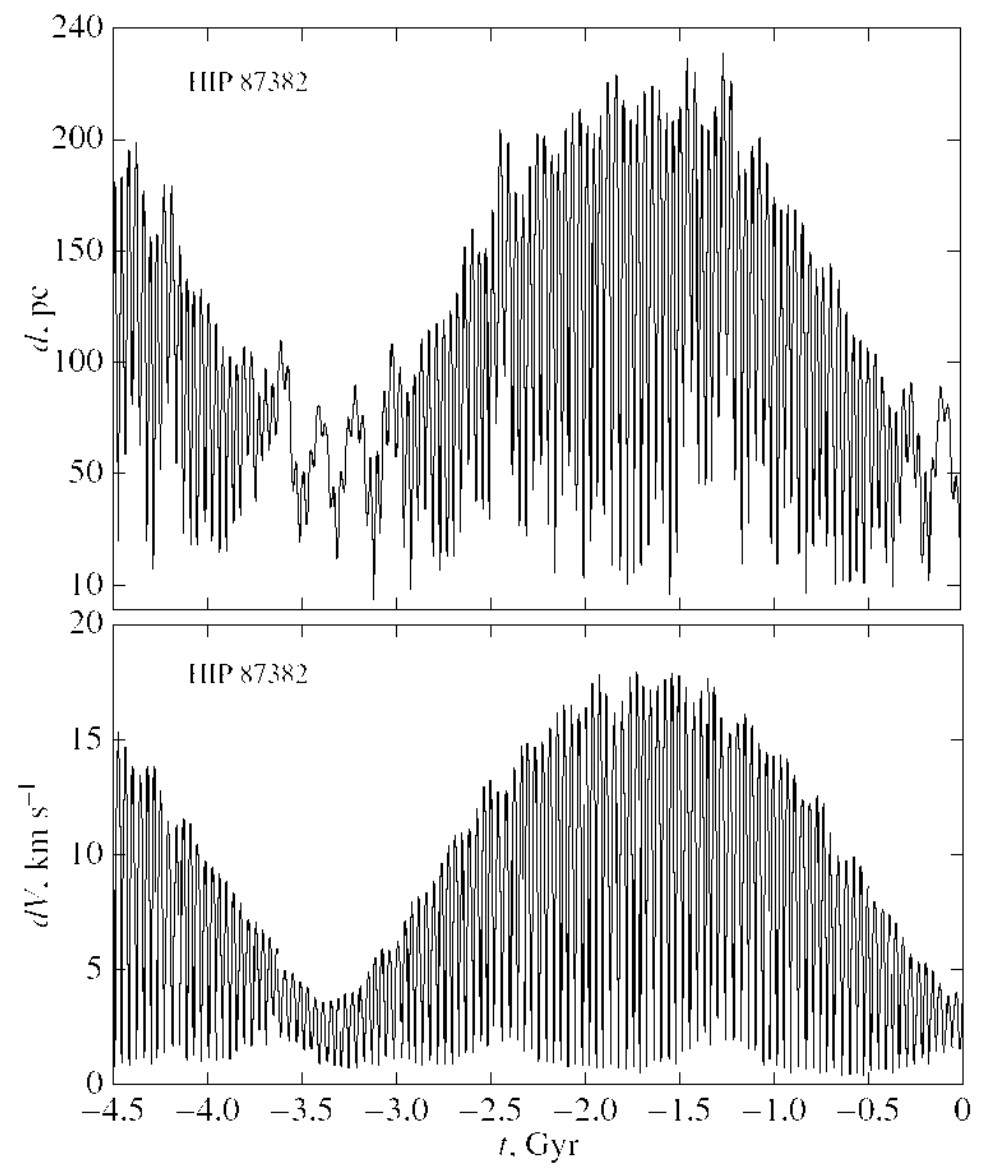

Figure 4: Parameters $d$ and $d V$ of the encounter between HIP 87382 and the solar orbit versus time for the two-armed spiral pattern.

\section{Statistical Simulations}

We calculated the encounter parameters by taking into account the random errors. The errors in the Suns peculiar velocity components $\left(U_{\odot}, V_{\odot}, W_{\odot}\right)_{L S R}$ were taken to be $(0.5,1,0.3) \mathrm{km} \mathrm{s}^{-1}$, a $10 \%$ level in the initial velocities $(U, V, W)$ and coordinates $(X, Y, Z)$ of the stars as well as in the parameters of the spiral density wave.

We performed our simulations for HIP 87382, because it has very small random errors in the initial coordinates and velocities. It turned out that there are interesting results in our simulations even in a symmetric potential (mainly due to the variations in the Suns peculiar velocity). Therefore, we present the results for HIP 87382 in the time interval from -4.5 to -2.5 Gyr for two cases: (1) the orbits were constructed only in a symmetric potential and (2) with the influence of the spiral density wave added:

1. For $d<10 \mathrm{pc},|d V|<10 \mathrm{~km} \mathrm{~s}^{-1}, p=0.0002(0.02 \%)$, for $d<10 \mathrm{pc},|d V|<5 \mathrm{~km}$ $\mathrm{s}^{-1}, p=0.0001(0.01 \%)$.

2. For $d<10 \mathrm{pc},|d V|<10 \mathrm{~km} \mathrm{~s}^{-1}, p=0.000009(0.0009 \%)$, for $d<10 \mathrm{pc}$, $|d V|<5 \mathrm{~km} \mathrm{~s}^{-1}, p=0.000006(0.0006 \%)$.

We see that $p$ decreased by less than one order of magnitude in case 1 and by two 


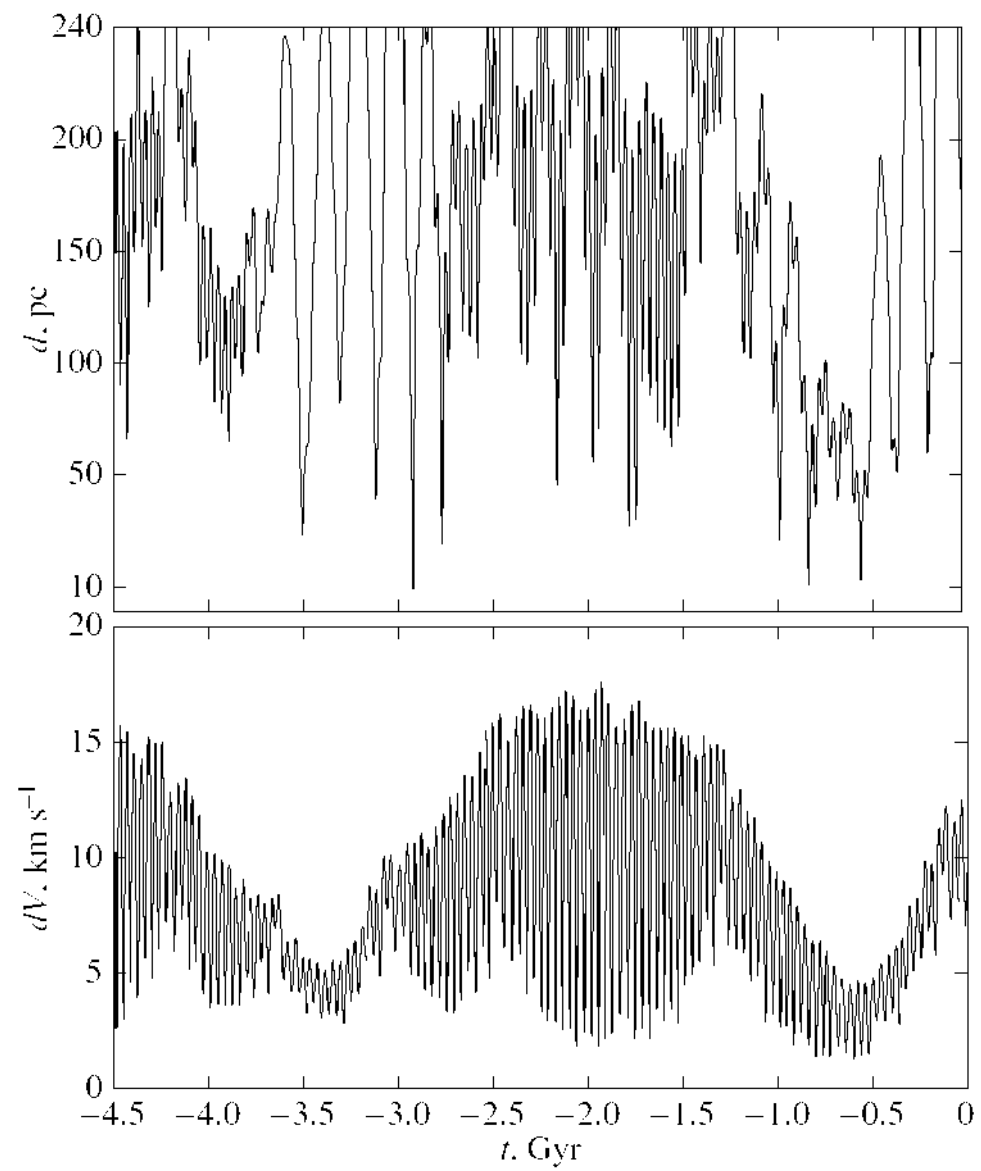

Figure 5: Parameters $d$ and $d V$ of the mutual encounters between HIP 47399 and HIP 87382 versus time for the two-armed spiral pattern.

orders of magnitude in case 2 compared to the analogous results described in the preceding section for this star. Obviously, the uncertainty in the parameters of the spiral density wave has a decisive influence.

One of the most important parameters in our model of the spiral wave is the ratio of the radial component of the perturbation from the spiral arms to the Galaxys total attraction, $f_{r 0}$. To investigate the robustness (stability to small perturbations) of our results, we determined the encounter parameters for $f_{r 0}$ from the range $0.04-0.07$ (with all of the remaining model parameters being fixed). The results are presented in Table 3. We see from this table that the results remain acceptable for our problem up to $f_{r 0}=0.065$ (the perturbation is $30 \%$ with respect to 0.05 ).

\section{Other Spiral-Structure Models}

The four-armed spiral pattern. For the four-armed spiral pattern, we set $m=4$ and the pitch angle $i=-10^{\circ}$. The remaining parameters were the same as those for the two-armed model, namely $\Omega_{p}=20 \mathrm{~km} \mathrm{~s}^{-1} \mathrm{kpc}^{-1}, f_{r 0}=0.05$, and $\chi_{\odot}=-117^{\circ}$. The 
Table 3: Encounter parameters calculated using the two-armed $(m=2)$ spiral pattern at various $f_{r 0}$

\begin{tabular}{|c|c|c|c|c|c|}
\hline HIP & $f_{r 0}$ & & $d_{\text {min }}, \mathrm{pc}$ & $t_{\text {min }}, \mathrm{Gyr}$ & $d V_{\min }, \mathrm{km} \mathrm{s}^{-1}$ \\
\hline 47399 & 0.045 & $*$ & 10 & -2.3 & 3 \\
& 0.050 & & 1 & -3.5 & 2 \\
& 0.055 & $*$ & 11 & -2.7 & 3 \\
& 0.060 & $*$ & 31 & -3.9 & 9 \\
& 0.065 & & 27 & -2.9 & 7 \\
\hline 87382 & 0.045 & $*$ & 6 & -3.3 & 4 \\
& 0.050 & & 4 & -3.1 & 6 \\
& 0.055 & & 2 & -2.1 & 17 \\
& 0.060 & & 5 & -2.2 & 16 \\
& 0.065 & $*$ & 26 & -3.5 & 4 \\
\hline
\end{tabular}

Note. ${ }^{*}$ - for the local minimum from the interval $t<-2$ Gyr.

simulation results are presented in Figs. 6 and 7.

We can seen from comparison of Figs. 6 and 3 that the four-armed model of the spiral pattern for HIP 47399 yields more interesting results: at $t \approx 4 \mathrm{Gyr}$, a global minimum is observed in encounters to distances of less than $30 \mathrm{pc}$ with relative encounter velocities $<2 \mathrm{~km} \mathrm{~s}^{-1}$.

As can be seen from comparison of Figs. 7 and 4, the picture of encounters with the solar orbit did not change qualitatively for HIP 87382.

The $2+4$ spiral pattern. For the composite $(2+4)$ model of the spiral pattern (Lépine et al. 2001; Mishurov and Acharova 2010), the spiral wave potential (6) contains two terms with amplitudes $A_{2}$ (two-armed component) and $A_{4}$ (four-armed component). In this model, the Sun is very close to the corotation circle and, as Mishurov and Acharova (2010) showed, the influence of the spiral structure is so strong that the test model particles are scattered over a significant spatial volume.

The following model parameters were adopted. For the two-armed component: $m=$ $2, i_{2}=-7^{\circ}, \chi_{\odot}=300^{\circ}$. For the four-armed component: $m=4, i_{4}=-14^{\circ}, \chi_{\odot}=135^{\circ}$. Both spiral patterns rotate with the same angular velocity $\Omega_{p}=\Omega_{0}$. In our case, $\Omega_{0}=220 / 8.5=25.9 \mathrm{~km} \mathrm{~s}^{-1} \mathrm{kpc}^{-1}$, as follows from the Allen-Santillan (1991) model parameters. We took $f_{r 0}=0.05$ when calculating $A_{2}$ and used $A_{2} / A_{4}=0.8$ to determine $A_{4}$.

The results are presented in Figs. 8 and 9. We can see that for both stars, HIP 47399 and HIP 87382, there are encounters of interest to us.

\section{CONCLUSIONS}

Based on the proposed kinematic approach to searching for the Sun's siblings from a common cluster, we selected $162 \mathrm{~F}, \mathrm{G}$, and K stars from the Hipparcos catalog with low heliocentric space velocities $\left(<8 \mathrm{~km} \mathrm{~s}^{-1}\right)$ from a solar neighborhood $\approx 200 \mathrm{pc}$ in radius. 

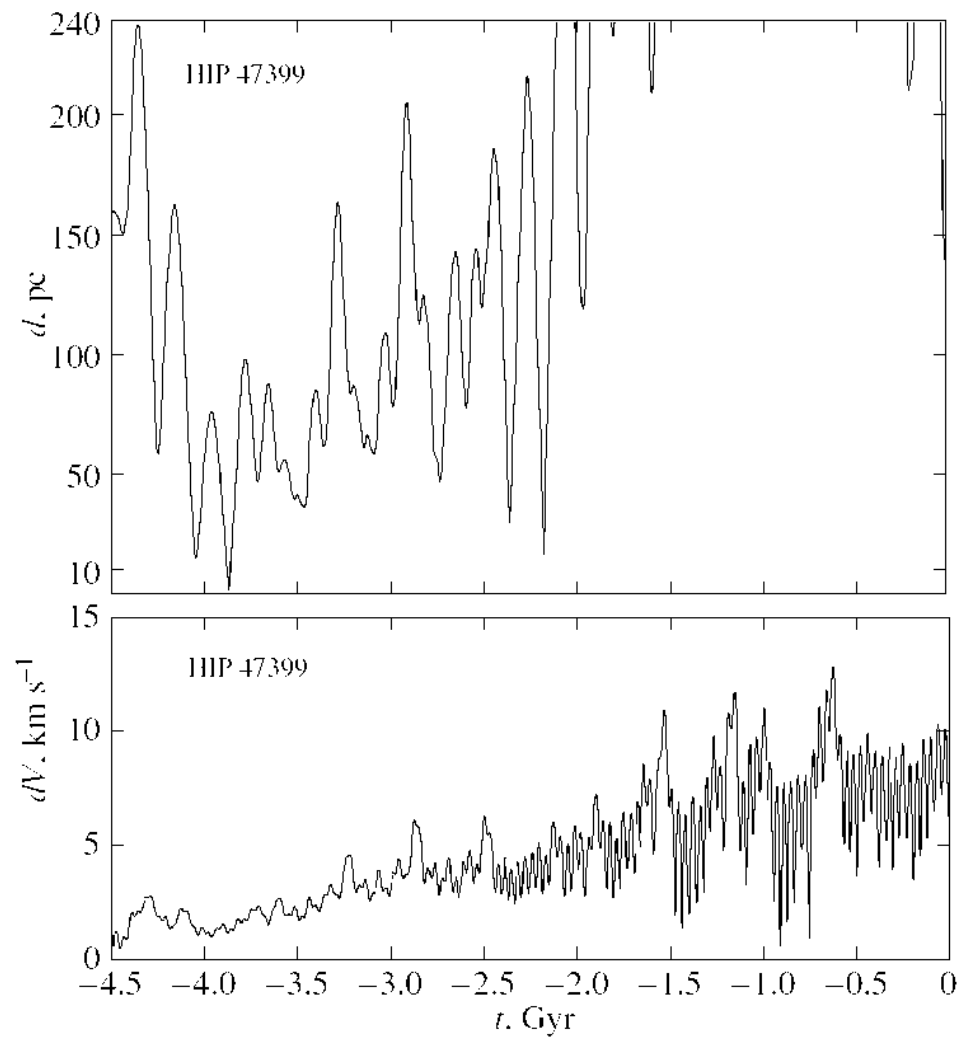

Figure 6: Parameters $d$ and $d V$ of the encounter between HIP 47399 and the solar orbit versus time for the four-armed spiral pattern.

For all these stars, we constructed the Galactic orbits for 4.5 Gyr into the past using the Allen-Santillan (1991) axisymmetric model Galactic potential that additionally included the perturbations from the spiral density wave. The parameters of the encounter with the solar orbit were calculated for each orbit.

We confirmed the conclusion reached by Mishurov and Acharova (2010) that the spiral density waves have a significant influence on the search results.

We considered the influence of a stationary spiral structure for the two- and fourarmed models $m=2,4$ as well as the composite $2+4$ model (Lépine et al. 2001) and found that they all confirm the results of our search.

We found that almost all of the stars considered in the past receded from the solar orbit fairly rapidly and far. Two single stars, HIP 87382 and HIP 47399, constitute an exception. In the two-armed model of the Galactic spiral pattern, their orbits oscillate near the solar orbit with an amplitude of $\approx 250 \mathrm{pc}$; there are peak close encounters to distances $d<10 \mathrm{pc}$; both stars have a wide minimum up to distances $d<60 \mathrm{pc}$ in the time interval $-4 \div-3$ Myr consistent with their age estimates. HIP 47399 is interesting in that its heliocentric velocity is always low, while this velocity in the time interval $-4 \div-3$ Myr is $|d V|<3 \mathrm{~km} \mathrm{~s}^{-1}$. The closest and longest encounters at $t \approx-4$ Gyr are observed for the four-armed model $(m=4)$ for HIP 47399 (Fig. 6).

As a result, we conclude that HIP 47399 and HIP 87382 are of considerable interest 


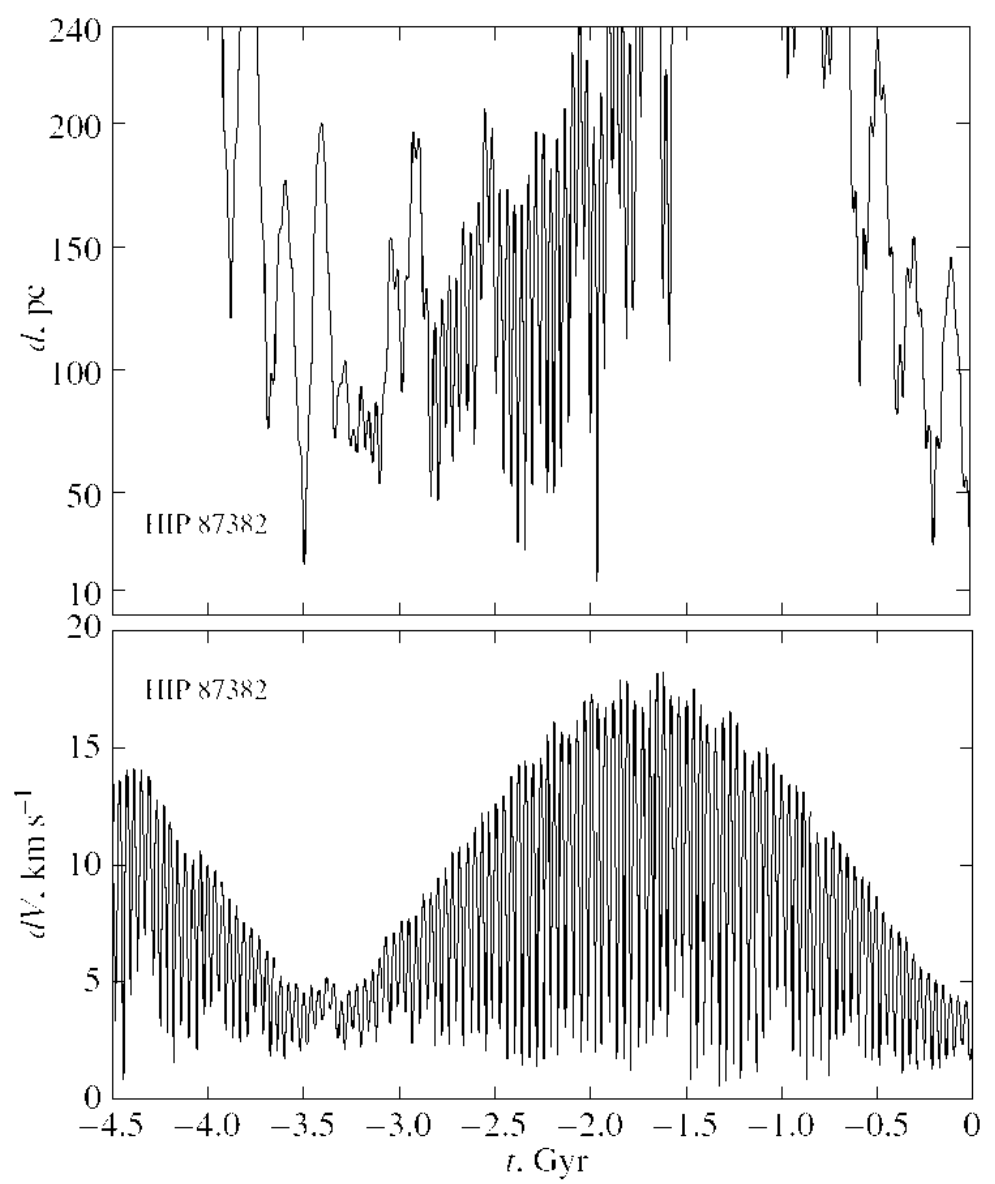

Figure 7: Parameters $d$ and $d V$ of the encounter between HIP 87382 and the solar orbit versus time for the four-armed spiral pattern.

as possible candidates for the Sun's siblings from the hypothetical parent cluster.

\section{ACKNOWLEDGMENTS}

We are grateful to the referees for helpful remarks that contributed to an improvement of the paper. We separately thank Yu. N. Mishurov for the discussion of the problems considered here. The SIMBAD search database provided a great help to our study. This work was supported by the Russian Foundation for Basic Research (project no. 08-020040) and in part by the "Origin and Evolution of Stars and Galaxies" Program of the Presidium of the Russian Academy of Sciences and the Program of State Support for Leading Scientific Schools of the Russian Federation (project. NSh-3645.2010.2, "Multiwavelength Astrophysical Studies").

\section{REFERENCES}

1. I.A. Acharova, J.R.D. Lépine, Yu.N. Mishurov, et al., MNRAS 402, 1149 (2010).

2. C. Allen and A. Santillan, Rev. Mex. Astron. Astrofis. 22, 255 (1991). 


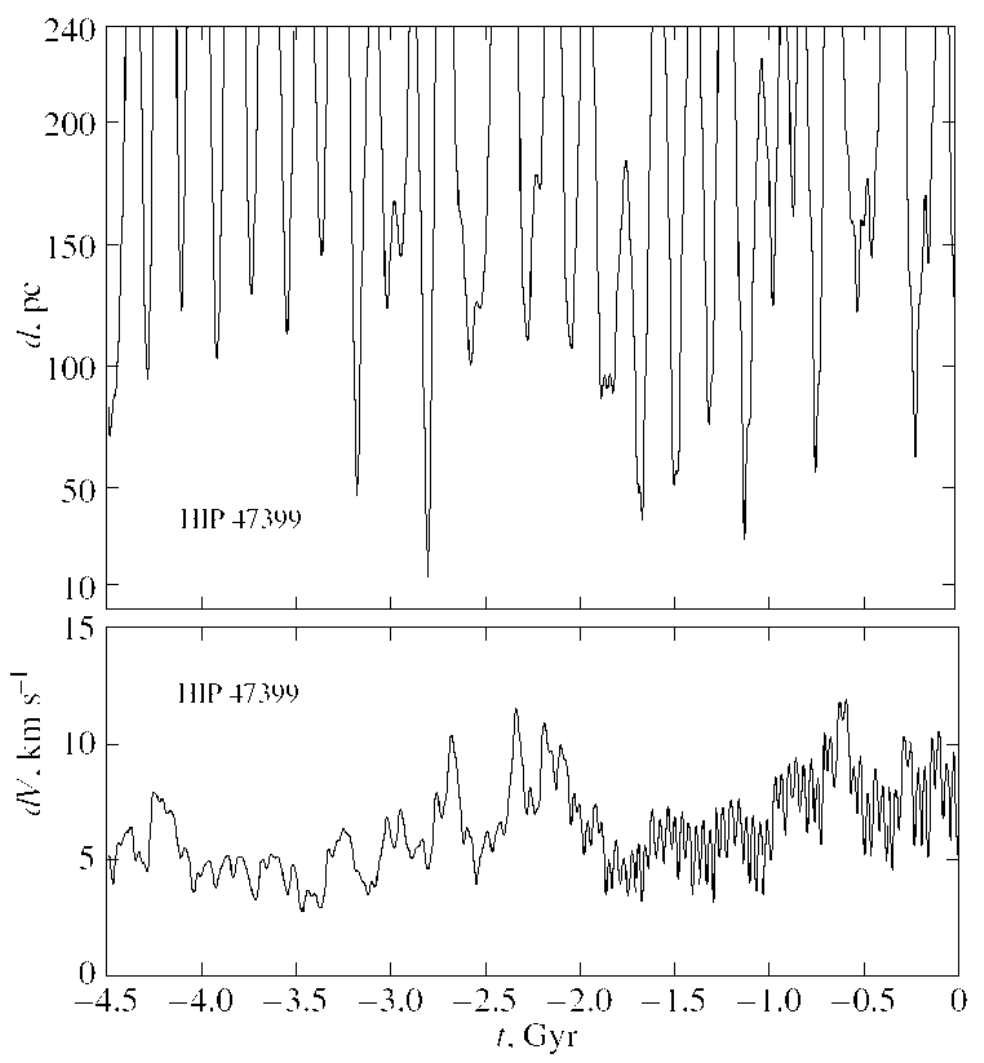

Figure 8: Parameters $d$ and $d V$ of the encounter between HIP 47399 and the solar orbit versus time for the composite $(2+4)$ spiral pattern. 


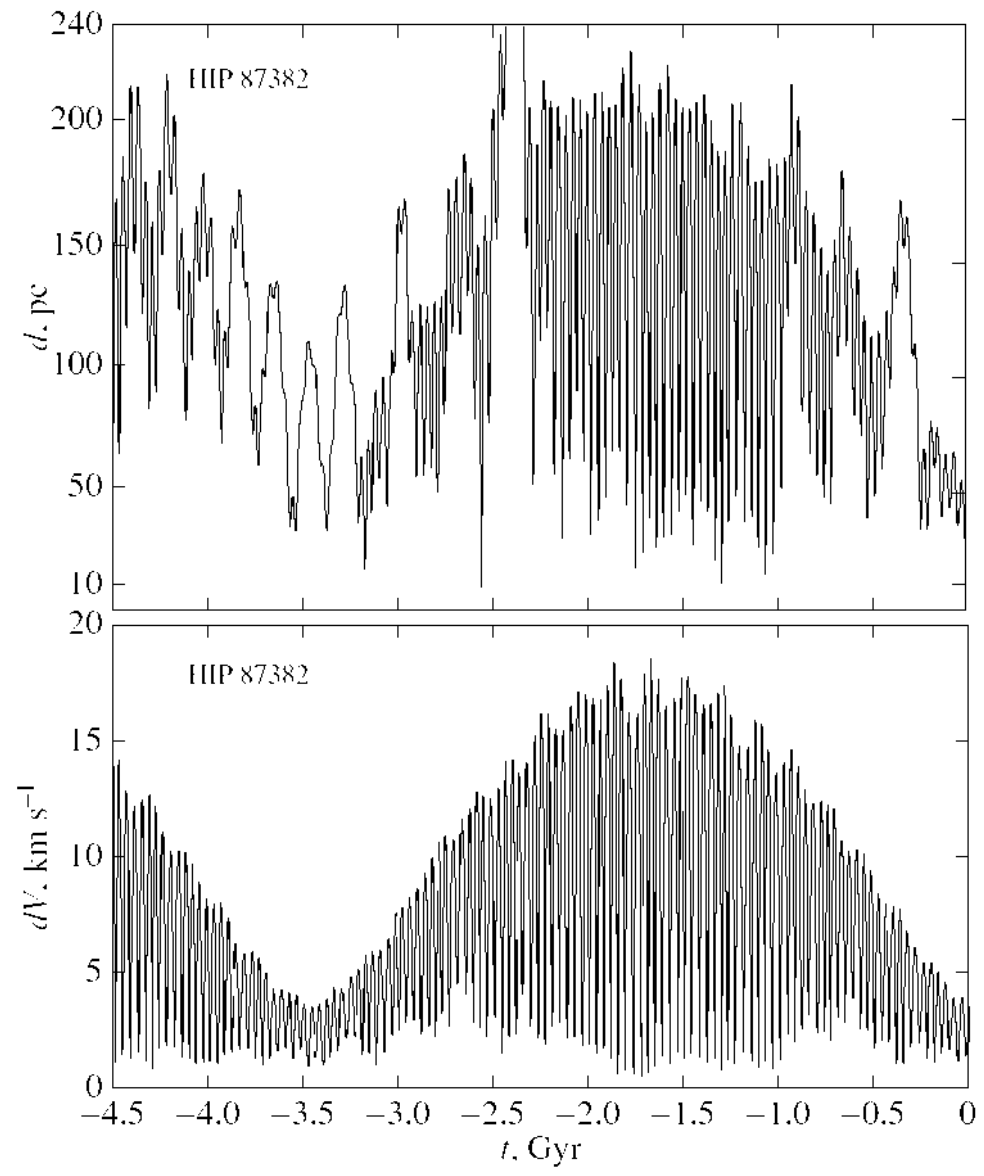

Figure 9: Parameters $d$ and $d V$ of the encounter between HIP 87382 and the solar orbit versus time for the composite $(2+4)$ spiral pattern. 
3. J. Baba, Y. Asaki, J. Makino, et al., Astrophys. J. 706, 471 (2009).

4. J.J. Binney, MNRAS 401, 2318 (2010).

5. J. Bland-Hawthorn and K. Freeman, Publ. Astron. Soc. Austral. 21, 110 (2004).

6. J. Bland-Hawthorn, M.R. Krumholz, and K. Freeman, Astrophys. J. 713, 166 (2010).

7. V.V. Bobylev, A.T. Bajkova, and A.S. Stepanishchev, Astron. Lett. 34, 515 (2008).

8. V.V. Bobylev, A.T. Bajkova, and A.A. Mylläri, Astron. Lett. 36, 27 (2010).

9. V.V.Bobylev, and A.T. Bajkova, MNRAS 408, 1788 (2010).

10. A.G.A. Brown, S.F. Portegies Zwart, and J. Bean, MNRAS 407, 458 (2010).

11. Ya.O. Chumak, A.S. Rastorguev, and S.D. Aarseth, Astron. Lett. 31, 308 (2005).

12. Ya.O. Chumak and A.S. Rastorguev, Astron. Lett. 37, 157 (2011).

13. Ya.O. Chumak and A.S. Rastorguev, Astron. Lett. 34, 446 (2008).

14. V.P. Debattista, O. Gerhard, and M. N. Sevenster, MNRAS 334, 355 (2002).

15. P. Demarque, J.Woo, Y.Kim, and S.K. Yi, Astrophys. J. Suppl. Ser. 155, 667 (2004).

16. P. Englmaier, M. Pohl, and N. Bissantz, arXiv astroph: 0812.3491 (2008).

17. D. Fernandez, F. Figueras, and J. Torra, Astron. Astrophys. 372, 833 (2001).

18. D. Fernandez, F. Figueras, and J. Torra, Astron. Astrophys. 480, 735 (2008).

19. O. Gerhard, Memorie della Societa Astronomica Italiana Supplement, v.18, p.185 (2011).

20. G.A. Gontcharov, Astron. Lett. 32, 759 (2006).

21. J. Holmberg, B. Nordström, and J. Andersen, Astron. Astrophys. 501, 941 (2009).

22. L.G. Hou, J.L. Han, and W.B. Shi, Astron. Astrophys. 499, 473 (2009).

23. Y.C. Joshi, MNRAS 378, 768 (2007).

24. A.H.W. Küpper, A. Macleod, and D.C. Heggie, MNRAS 387, 1248 (2008).

25. F. van Leeuwen, Astron. Astrophys. 474, 653 (2007).

26. J.R.D. Lépine, Yu.N. Mishurov, and S.Yu. Dedikov, Astrophys. J. 546, 234 (2001).

27. C.C. Lin and F.H. Shu, Astrophys. J. 140, 646 (1964).

28. C.C. Lin, C. Yuan, and F.H. Shu, Astrophys. J. 155, 721 (1969).

29. A.M. Melnik and P. Rautiainen, Astron. Lett. 35, 609 (2009).

30. I. Minchev and B. Famaey, Astrophys. J. 722, 112 (2010).

31. Yu.N. Mishurov and I.A. Zenina, Astron. Astrophys. 341, 81 (1999).

32. Yu.N. Mishurov and I.A. Acharova, MNRAS 412, 1771 (2011).

33. S. Naoz and N. J. Shaviv, New Astron. 12, 410 (2007).

34. L. Pompéia, T. Masseron, B. Famaey, et al., arXiv astro-ph:1101.2583 (2011).

35. M.E. Popova and A.V. Loktin, Astron. Lett. 31, 171 (2005).

36. S.F. Portegies Zwart, Astrophys. J. 696, L13 (2009).

37. S.E. Robinson, S.M. Ammons, K.A. Kretke, et al., Astrophys. J. Suppl. Ser. 169, 430 (2007).

38. D. Russeil, Astron. Astrophys. 397, 134 (2003).

39. R. Schönrich, J. Binney, and W. Dehnen, MNRAS 403, 1829 (2010).

40. J.A. Sellwood and J.J. Binney, MNRAS 336, 785 (2002).

41. I.I. Shevchenko, Astrophys. J. 733, 39 (2011).

42. G. Takeda, E.B. Ford, A. Sills, et al., Astrophys. J. Suppl. Ser. 168, 297 (2007).

43. The HIPPARCOS and Tycho Catalogues, ESA SP-1200 (1997).

44. M. Valtonen, P. Nurmi, J.-Q. Zheng, et al., Astrophys. J. 690, 210 (2009).

45. R. Wielen, B. Fuchs, and C. Dettbarn, Astron. Astrophys. 314, 438 (1996).

46. J. Williams, Contemp. Phys. 51, 381 (2010).

47. C. Yuan, Astrophys. J. 158, 889 (1969). 